\title{
HIGHLIGHTS
}

REPRODUCTIVE ENDOCRINOLOGY

\section{Polycystic ovary syndrome: simvastatin and metformin compared}

Simvastatin is superior to metformin for the treatment of women with polycystic ovary syndrome (PCOS), according to a new study.

"Over the last decade, metformin became a commonly used drug in the treatment of PCOS. Since metformin is thought to act, at least in large part, via reduction of hyperinsulinemia, while statins actions were expected to be related to anti-inflammatory properties, we proposed that a combination of statins and metformin might have an additive beneficial effect on PCOS," explains Antoni J. Duleba of the University of California Davis. "Unexpectedly, a combination of metformin and statin was not superior to the use of statin alone."
The researchers randomly allocated 136 young women with PCOS to receive simvastatin, metformin, or simvastatin plus metformin for 12 weeks. The women, most of whom were lean, fulfilled the Rotterdam criteria of PCOS and were also hyperandrogenic.

Reduction in testosterone levels, and improvements in markers of systemic inflammation and endothelial function and in clinical hyperandrogenism were observed in all three groups. By contrast, improvements in lipid profile and insulin sensitivity and reductions in levels of DHEAS only occurred in women in the two groups who received simvastatin.

The researchers are now evaluating the direct effects of statins on the ovaries.

"It is likely that statins will be used in the

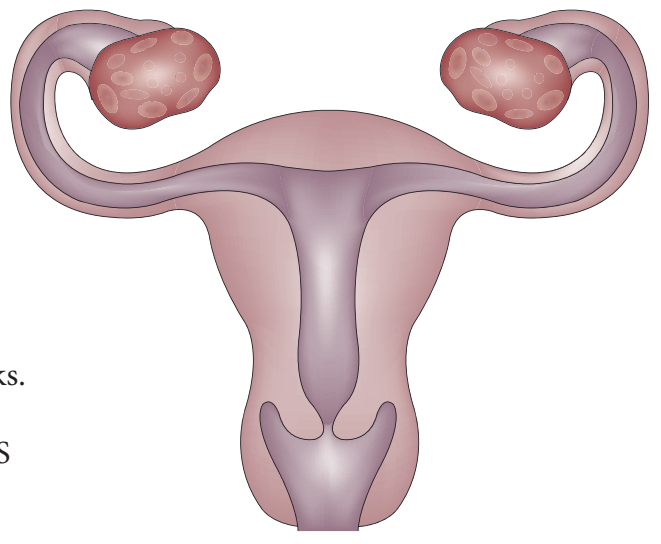

treatment of PCOS, especially for women not planning pregnancy but interested in improvement of long-term cardiovascular health," concludes Duleba.

\section{Carol Wilson}

Original article Banaszewska, B. et al. Comparison of simvastatin and metformin in treatment of polycystic ovary syndrome: prospective randomized trial. J. Clin. Endocrinol. Metab. 94, 4938-4945 (2009) 